\title{
Mesoscopic simulations of phase transitions in lipid bilayers
}

\author{
Marieke Kranenburg, Christophe Laforge and Berend Smit* \\ Department of Chemical Engineering, University of Amsterdam, Nieuwe Achtergracht 166 1018, \\ WV Amsterdam, The Netherlands.E-mail:b.smit@science.uva.nl
}

\begin{abstract}
The formation of the rippled phase in biological membranes and its relation with anomalous swelling are still lacking a molecular explanation. Starting from all-atom simulations we use a mapping to create a mesoscopic model of the lipid dimyristoylphosphatidylcholine (DMPC) in water. We use this model to study the phase behaviour of lipid bilayers. Depending on the lipid structure and head group, our simulations reproduce the experimental phase diagrams. The anomalous swelling is caused by conformational changes of the lipid tails but is not directly related to the rippled phase. A key factor for the rippled phase is a frustration between the surface area of the heads and the lateral density of the tails.
\end{abstract}

Phospholipids can self-assemble in water to form bilayer structures, which are seen as model systems of biological membranes. ${ }^{1}$ At high temperatures lipids form a flat fluid membrane $\left(\mathrm{L}_{\alpha}\right.$ phase $)$ and at low temperatures a flat gel phase in which the lipid tails are ordered and tilted $\left(\mathrm{L}_{\beta^{\prime}}\right.$ phase). For certain phospholipids between the $\mathrm{L}_{\alpha}$ and $\mathrm{L}_{\beta^{\prime}}$ phase phases a stable supramolecular periodic structure was observed ${ }^{2-4}$ which is characterized by a long-wavelength rippling of the bilayer ( $\mathrm{P}_{\beta^{\prime}}$ phase) and a (anomalous) swelling of the membrane. This rippled phase has attracted the attention of many groups, ${ }^{2-7}$ but still lacks a molecular understanding. ${ }^{1,8}$ Using state of the art molecular dynamics simulations, it is possible to obtain detailed structural information of a single phase, for example, the gel phase, ${ }^{9}$ but these are too time consuming to determine a complete phase diagram. In this work, we use an alternative approach in which realistic all-atom simulations are used to determine the effective intramolecular interaction parameters of a mesoscopic model. At this mesoscopic level simulations are four to five orders of magnitude more efficient, ${ }^{10,11}$ allowing us to compute complete phase diagrams.

In a dissipative particle dynamics (DPD) simulation a particle represents the centre of mass of a cluster of atoms. The total force on a particle consists of dissipative, random, and conservative forces. ${ }^{12,13}$ For the conservative force we use a soft-repulsive interaction

$$
\boldsymbol{F}_{i j}^{\mathrm{C}}=\left\{\begin{array}{cc}
a_{i j}\left(1-r_{i j} / r_{\mathrm{c}}\right) \hat{\boldsymbol{r}}_{i j} & \left(r_{i j}<r_{\mathrm{c}}\right) \\
0 & \left(r_{i j} \geq r_{\mathrm{c}}\right)
\end{array}\right.
$$

where $r_{i j}$ is the distance between particles $i$ and $j, a_{i j}$ is the parameter characterising the interaction between two particles, and $r_{\mathrm{c}}$ is the cut-off radius. In our mesoscopic model, we distinguish three types of particles, $w, h$, and to mimic the water and the head- and tail-atoms of a lipid, respectively.

A coarse graining procedure to map the interactions of realistic molecules on DPD interaction parameters has been developed by Groot and Warren. ${ }^{14}$ In this procedure the value of the repulsion parameter is taken such that the DPD waterlike particles reproduce the compressibility of water. The interactions of the hydrophilic and hydrophobic particles $\left(a_{\mathrm{ww}}=a_{\mathrm{tt}}=25, a_{\mathrm{ht}}=a_{\mathrm{wt}}=80\right.$, and $\left.a_{\mathrm{hw}}=15\right)$ are based on the Flory-Huggins solubility parameters. We vary the headhead $\left(a_{\mathrm{hh}}\right)$ interaction parameter to study the effect of changing the interactions between the head groups of a lipid, mimicking changes in the chemical nature of the head group or adding salt to the system.

We divided the phospholipid dimyristoylphosphatidylcholine (DMPC) into equal volumes of $90 \AA^{3}$, using the phospholipid component volumes determined by Armen et al. ${ }^{15}$ The mapping of the lipid results in a model consisting of three hydrophilic head beads and two tails, each consisting of five hydrophobic tail beads. Two consecutive beads are connected by harmonic springs with spring constant $k_{r}=100$ and equilibrium distance $r_{0}=0.7$. When we only use a harmonic spring to connect the lipid beads, we do not reproduce the chain length dependence of the area per lipid in a membrane in the $\mathrm{L}_{\alpha}$ phase correctly. For a correct description of this dependence it is important to introduce a more realistic description of the intramolecular interactions, for which we have used a coarse-graining procedure. In this procedure we performed molecular dynamics simulations of a single phospholipid in water using a realistic all-atom representation, using the CharmM force field for DMPC and the TIP3P model for water. From these single lipid simulations we computed the distributions of the centers of mass associated with the coarse grained DPD beads. The parameters for the bond-bending interactions in the DPD model, $U(\theta)=1 / 2 k_{\theta}\left(\theta-\theta_{0}\right)^{2}$, were obtained by matching the corresponding distributions of the DPD particles. We added a bond-bending potential between three consecutive beads in the tails with bending constant $k_{\theta}=$ 6 and equilibrium angle $\theta=180^{\circ}$. An additional bond-bending potential is applied between the vectors connecting the tails to the head group, with $k_{\beta}=3$ and $\theta_{0}=90^{\circ}$. With these parameters our simulations predict an area per surfactant of $69 \AA^{2}$ of the membrane in the $\mathrm{L}_{\alpha}$ or fluid phase, independent of the chain length, which is in good agreement with the experimental values which are in the range $58-72 \AA^{2}$. More details can be found in ref. 16 .

A biological membrane is not subject to external constraints and therefore adopts a configuration which is tensionless. In a simulation in which the total area and number of lipid molecules are fixed, the resulting membrane has a non-zero interfacial tension. Lipowski and coworkers ${ }^{17}$ emphasize the importance of simulating at exactly the area for which the interfacial tension is zero and determine this area iteratively. We use a different approach in which we simulate an ensemble in which we can impose the interfacial tension. After a randomly selected number of DPD steps we perform a Monte Carlo move in which we change the area of our bilayer in such a way that the total volume of the system remains constant. ${ }^{18}$ To obtain the tensionless state of the bilayer $\gamma$ is set to zero. The importance of this method is that it allows us to observe directly phase transitions in which the area per lipid changes. 
We have used a system of 800 lipids with $8000-15,000$ water particles to ensure a fully hydrated bilayer with overall density $\rho=3$. A typical simulation required 100,000 cycles, which consists of, on average, 150 DPD time steps and one Monte Carlo attempt to change the area. The first 20,000 cycles are equilibration. The equations of motion are integrated with a modified velocity Verlet algorithm with $\Delta t=0.03$. We use reduced units with $r_{\mathrm{c}}$ as the unit of length, the mass of a particle $m$ as the unit of mass, and $a_{\mathrm{ww}}$ as unit of energy. From this coarse-graining procedure, the interaction parameters are defined in units of $k_{\mathrm{B}} T$. To use reduced units, we define $k_{\mathrm{B}} T_{0}=1$ where $T_{0}$ is room temperature. The $a_{\mathrm{ww}}$ parameter has been fitted to give the correct compressibility of water at room temperature and at the assumed density. In principle, we could use the same procedure to match the compressibility of water at different temperatures. This gives, however, a temperature dependent $a$ parameter which would make the interpretation of our results more complex. Therefore we have chosen to keep the parameters fixed and only change the temperature. In the following we will use the notation $T^{*}$ to indicate the reduced temperature.

Fig. 1 shows the computed phase diagrams for lipids with different tail lengths as a function of temperature and the headgroup interaction. At high temperatures the $\mathrm{L}_{\alpha}$ phase is stable; the tails are disordered and have no preferred tilt angle. At low temperatures we find the $\mathrm{L}_{\mathrm{c}}$ phase in which the tails are ordered and tilted. Also the heads show some order in this phase. The stability of this phase is determined by the tails, which pack optimally in a tilted configuration. Depending on the headgroup interactions the configuration of the heads, however, can be far from optimal. At higher temperatures the head group interactions determine whether the system will form the $\mathrm{L}_{\beta}$ (flat gel) phase or the $\mathrm{L}_{\beta^{\prime}}$ (tilted gel) phase. For sufficiently large values of $a_{\mathrm{hh}}$ the most favorable configuration for the heads is to be surrounded by water molecules (strong hydration), while for low values of $a_{\mathrm{hh}}$ head-head contacts are favorable and water is expelled from the head region. For low values of $a_{\mathrm{hh}}$ the flat gel phase $\left(\mathrm{L}_{\beta}\right)$ is stable; the area per lipid is relatively small, the tails are ordered but no tilt is present. For sufficiently
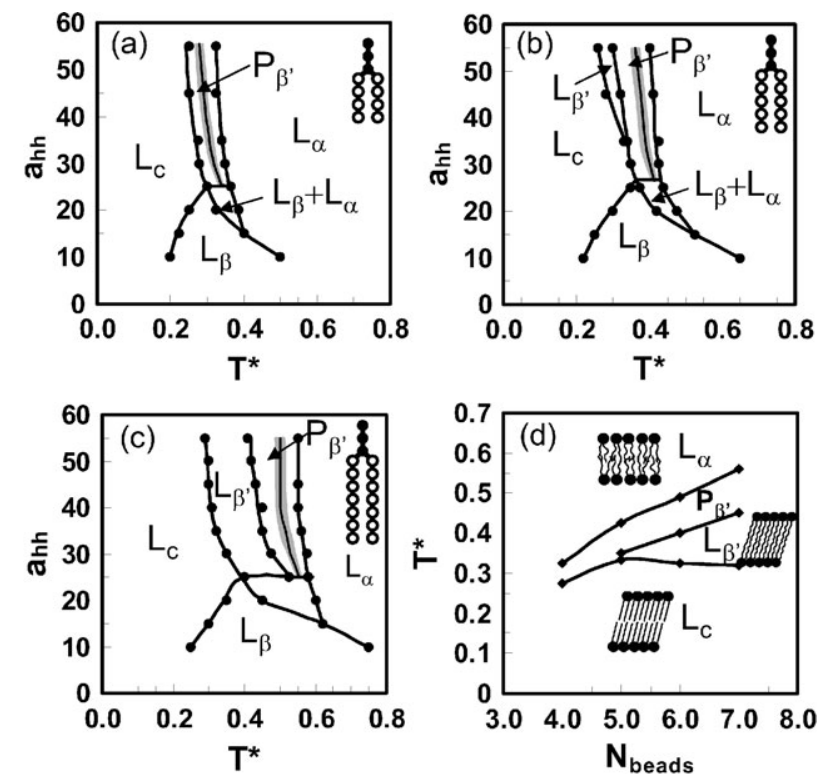

Fig. 1 Phase behavior as a function of the temperature and headgroup interactions for (a) $h_{3}\left(t_{4}\right)_{2}$, (b) $h_{3}\left(t_{5}\right)_{2}$, and (c) $h_{3}\left(t_{7}\right)_{2}$. The transition temperatures as a function of tail length for the typical head -head interactions $\left(a_{\mathrm{hh}}=35\right)$ are shown in $(\mathrm{d})$. As order parameters to characterize the phases we used order of the tails, tilt angle, and hydrophobic thickness. Jumps in these order parameters were used to locate the phase boundaries. The thin line in the $\mathrm{P}_{\beta^{\prime}}$ coexistence region corresponds to the condition of $50 \%-50 \%$ of both phases. In a narrow region around this line we find the rippled structure shown in Fig. 2. high $a_{\mathrm{hh}}$ the increase of the water hydration results in the $\mathrm{L}_{\beta^{\prime}}$ phase in which the tails partially lose their order but keep their tilt. The occurrence of this phase, however, depends on the tail length; for the long tails it is stable over a large temperature and range, while for the short tails this phase disappears. Interestingly, transition from $\mathrm{L}_{\mathrm{c}}$ or $\mathrm{L}_{\beta^{\prime}}$ to $\mathrm{L}_{\alpha}$ occurs via a narrow region. If we simulate exactly at the middle of this region where each phase occupies $50 \%$ of the space, the system forms the structure shown in Fig. 2, which indicates the onset of the rippled phase $\mathrm{P}_{\beta^{\prime}}$. Similar structures are found at $40 \%-$ $60 \%$ of the two phases, but we have not been able to locate the exact boundaries of this phase. Experimentally, the rippled phase concerns length scales that are much longer than we can access with our simulations. Therefore we cannot address questions related to the organization of these microdomains in space and how this organization is related to the thermal history of the sample. However, the similarities with the experimental phase diagram do suggest that the phase separation in microdomains indicates the onset of the rippled phase, while the boundary of the coexistence regions might be related to the experimentally observed pretransition.

If we increase the tail length, the period of the ripple increases. In some simulations the ripple was formed diagonally in the $y z$-plane of the simulation box. Since we apply periodic boundary conditions in all three directions, one might wonder if this is a rippled phase or just the formation of two domains. Increasing the system size to 1800 lipids shows that in this case the striped structure is again formed parallel to the $y$ axis of the system, indicating that the rippled phase is the stable phase. Once optimized the period of the ripple, there is a linear relation between the system size and the number of ripples. In the region between the $L_{\beta}$ and $L_{\alpha}$ phases we do not find any indication of a rippled phase. If we take the typical head-head interaction used by Groot, $a_{\mathrm{hh}}=35$, we obtain the transition temperatures shown in Fig. 1(d), which nicely resembles the experimental phase diagrams. ${ }^{19,20}$ We repeated the simulations with the parameter set published by Groot and Rabone ${ }^{10}$ giving qualitatively similar phase diagrams but shifted to higher temperatures.

Our simulations show that a key factor in the formation of the rippled phase is the head group interaction. By decreasing this parameter the $L_{\beta}$ phase, in which the tails are not tilted, becomes stable and as a consequence the rippled phase is not formed with increasing temperature. Experimentally this scenario has been observed by decreasing the size of the lipid head group. ${ }^{8}$

The rippled phase has also attracted the attention because of the anomalous or critical swelling that was observed. This swelling was considered a key factor in understanding the rippled phase. However, it is still controversial what causes this swelling. ${ }^{1,21}$ Theories can be found that explain this
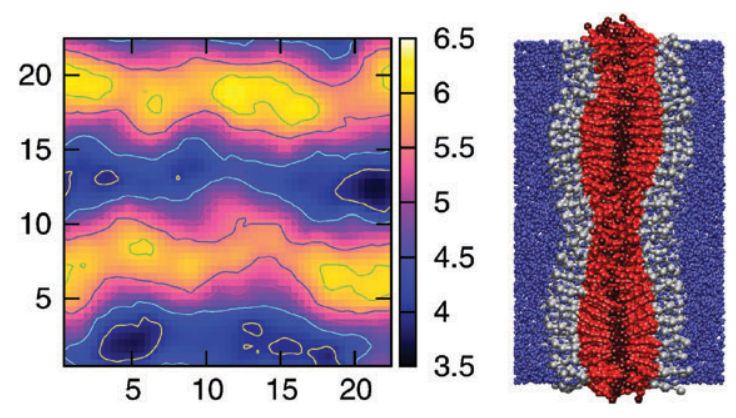

Fig. 2 Rippled phase for $\mathrm{h}_{3}\left(t_{4}\right)_{2}$ lipids $\left(a_{\mathrm{hh}}=45, T^{*}=0.3\right)$. At exactly equal amounts of material in the $\mathrm{L}_{c}$ and $\mathrm{L}_{\alpha}$ phases. Left: thickness of the membrane as a function of the position in the plane where the color coding gives the hydrophobic thickness of the membrane (yellow $\mathrm{L}_{\mathrm{c}}$ and purple $\mathrm{L}_{\alpha}$ phase). Right: side view in which the water particles are blue, the head groups grey, and the tails red. The darker color red is used for the end segments. 
swelling in terms of Helfrich's undulations forces, ${ }^{22,23}$ increase in conformational order of the hydrocarbon chains, ${ }^{24,25}$ increase in the water thickness, ${ }^{1}$ or increase in head-group layer thickness. Moreover, the recent experimental data of Mason et al. ${ }^{8}$ show that anomalous swelling even occurs in systems that do not form a rippled phase.

Fig. 3 shows that in our simulations decreasing the temperature results in an increase of the bilayer thickness, because of an increase in the order of the tails. Depending on the head group interactions these swelling curves show a very different behavior, similar to what has been observed experimentally. ${ }^{8}$ For low values of the head-head interaction, $a_{\mathrm{hh}}<15$, we cross the $\mathrm{L}_{\alpha} \rightarrow \mathrm{L}_{\beta}$ transition and the thickness increases significantly and goes through a maximum at the transition $\mathrm{L}_{\beta} \rightarrow \mathrm{L}_{\mathrm{c}}$. In the $\mathrm{L}_{\mathrm{c}}$ phase the tails are tilted and therefore the membrane has a lower hydrophobic thickness. At these low temperatures the hydrophilic part of the lipid stretches which (partially) compensates this decrease. Since the driving force for the formation of the $L_{\beta}$ phase is the gain in energy if water is expelled from the head region, we observe that if we increase $a_{\mathrm{hh}}$ the maximum of the hydrophobic thickness decreases and at the triple point the maximum has disappeared. The temperature range for which the $\mathrm{L}_{\beta}$ phase is stable decreases and as a consequence we see a very sharp increase of the thickness which resembles the approach of a critical point. For those values of $a_{\mathrm{hh}}$ for which the $\mathrm{P}_{\beta^{\prime}}$ phase is observed, we observe a relatively strong increase of the thickness associated with the $\mathrm{L}_{\alpha} \rightarrow \mathrm{P}_{\beta^{\prime}}$ transition. Our simulations show that in all cases the (anomalous) swelling is simply the consequence of changes of the conformation of the hydrocarbon tails and is not directly related to the formation of the rippled phase.

The general picture of the rippled phase is that the shape is an asymmetric sawtooth, with a difference in thickness between the long and the short arm. ${ }^{2-4}$ However, the nature of the rippled phase is not yet clear since it is difficult to determine the structure of the two parts of the ripple experimentally. ${ }^{26}$ For example, Sun et l. $^{3}$ assume that the X-ray diffraction patterns are best fitted with a model in which asymmetry of the height profile is the dominant feature, while Sengupta et al. ${ }^{27}$ concluded that the difference in thickness of the two arms is the determining factor. Our simulations show that the two arms are two coexisting phases and therefore support the conclusion of Rappolt et al. ${ }^{28}$

A surprising aspect of the ripple phase is that, unlike ordinary coexistence, the system does not minimize the total interfacial area formed by the two phases. Our simulations show that the head-head interaction is a key factor in the formation of the ripple phase; if $a_{\mathrm{hh}}<25$ we do not observe the rippled phase. For $a_{\mathrm{hh}}>25$, the system can lower its

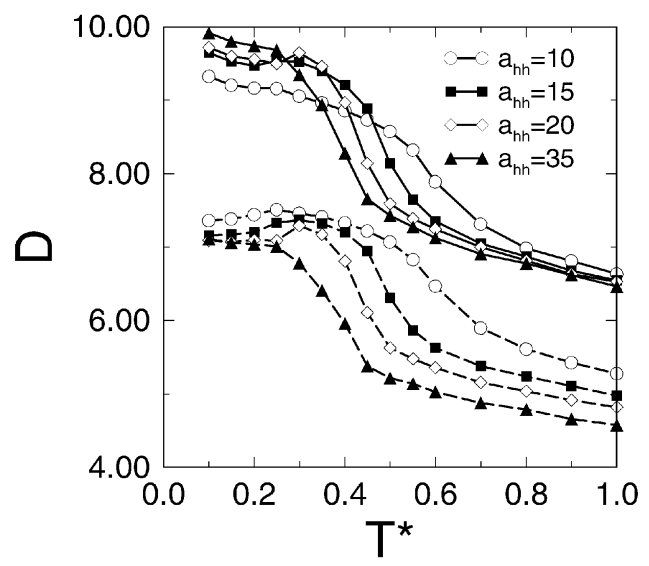

Fig. 3 Bilayer thickness (solid lines) and the hydrophobic thickness (dashed lines) as a function of the temperature for $h_{3}\left(t_{5}\right)_{2}$ lipids for various values of the head-head interactions.

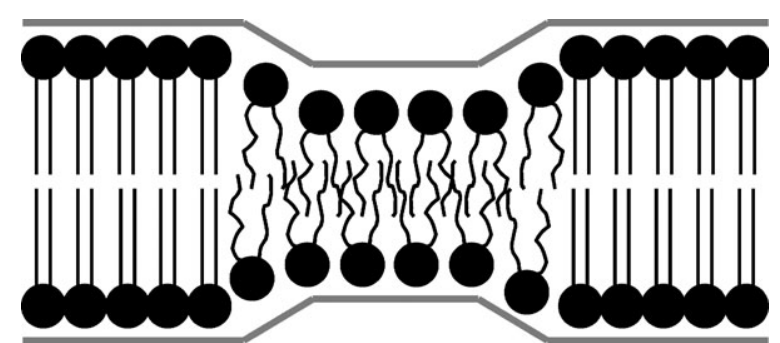

Fig. 4 Rippled phase; coexistence between the thick $\left(\mathrm{L}_{\mathrm{c}}\right.$ or $\left.\mathrm{L}_{\beta^{\prime}}\right)$ and thin $\left(\mathrm{L}_{\alpha}\right)$ phases.

energy if more head groups are exposed to water. In the coexistence region the head-group water contact area is locally increased (see Fig. 4), hence by increasing the number of interfaces the system can lower its energy. The total number of interfaces, however, will be limited by the repulsive forces between the ripples. The origin of this force is the elastic energy, which tends to minimize the curvature of the interface between the thick and thin parts. ${ }^{29}$ This aspect will depend on the tail length; the longer the tails the larger the difference in thickness that has to be crossed. This explains why we observe that the period of the ripple increases with tail length. Within the thick part of the ripple, the average orientation of the tilt is parallel to the direction of the ripple, which is important factor that stabilizes a linear interface.

It is interesting to compare these results with the continuum Landau theory, ${ }^{6}$ in which a rippled phase occurs due to the coupling of molecular tilt to the membrane curvature if the longitudinal elastic constant is negative. Our results show that microscopic origin of this negative constant is a surface area of the heads which is not compatible with the lateral density of the tails. Within the coexistence region we only observe the rippled structure in a narrow band around the line where we have approximately $50-50 \%$ material of both phases. This corresponds to the conditions in which, with the curvature constrain, the space filling problem leads to a striped (rippled) solution.

\section{Acknowledgements}

These investigations are supported by the Netherlands Research Council for Chemical Sciences (CW) and by the Netherlands Organization for Scientific Research (NWO) through PIONIER. We thank Daan Frenkel, Annalisa Fasolino, and John Nagle for their comments on our work.

\section{References}

1 J. F. Nagle and S. Tristram-Nagle, Biochim. Biophys. Acta, 2000, 1496, 159-195.

2 A. Tardieu, V. Luzzatti and F. C. Reman, J. Mol. Biol., 1973, 75, 711-733.

3 W.-J. Sun, S. Tristram-Nagel, R. M. Suter and J. F. Nagle, Proc. Natl. Acad. Sci. USA, 1996, 93, 7008-7012.

4 J. A. N. Zasadzinsky, J. Schneir, J. Gurley, V. Elings and P. K. Hansma, Science, 1988, 239, 1013-1015.

5 M. S. Falkovits, M. Seul, H. L. Frisch and H. M. McConnell, Proc. Natl. Acad. Sci. USA, 1982, 79, 3918-3921.

6 T. C. Lubensky and F. C. MacKintosh, Phys. Rev. Lett., 1993, 71, 1565-1568.

7 R. E. Goldstein and S. Leibler, Phys. Rev. Lett., 1988, 61, 22132216.

8 P. C. Mason, J. F. Nagle, R. M. Epand and J. Katsaras, Phys. Rev. E, 2001, 63, 30902.

9 H. L. Scott, Curr. Opin. Struct. Biol., 2002, 12, 495-502.

10 R. D. Groot and K. L. Rabone, Biophys. J., 2001, 81, 725-736.

11 J. C. Shelley, M. Y. Shelley, R. C. Reeder, S. Bandyopadhyay, P. B. Moore and M. L. Klein, J. Phys. Chem. B, 2001, 105, 97859792. 
12 P. J. Hoogerbrugge and J. M. V. A. Koelman, Europhys. Lett., 1992, 19, 155-160.

13 D. Frenkel and B. Smit, Understanding Molecular Simulations: from Algorithms to Applications, Academic Press, London, 2nd edn., 2002.

14 R. D. Groot and P. B. Warren, J. Chem. Phys., 1997, 107, 4423 4435

15 R. S. Armen, O. D. Uitto and S. E. Feller, Biophys. J., 1998, 75, 734-744.

16 M. Kranenburg, J.-P. Nicolas and B. Smit, Phys. Chem. Chem. Phys., 2004, 66, 4142-4151.

17 R. Goetz, G. Gompper and R. Lipowsky, Phys. Rev. Lett., 1999 82, 221-224.

18 M. Venturoli and B. Smit, PhysChemComm, 1999, 2, 45.

19 B. A. Cunningham, A.-D. Brown, D. H. Wolfe, W. P. Williams and A. Brain, Phys. Rev. E, 1998, 58, 3662-3672.

20 B. Tenchov, R. Koynova and G. Rapp, Biophys. J., 2001, 80, 1873-1890.
21 J. F. Nagle, H. I. Petrache, N. Gouliaev, S. Tristram-Nagle, Y. Liu, R. M. Suter and K. Gawrisch, Phys. Rev. E, 1998, 58, 7769-7776.

22 J. Lemmich, K. Mortensen, J. H. Ipsen, T. Honger, R. Bauer and O. G. Mouritsen, Phys. Rev. E, 1996, 53, 5169-5180.

23 S. Korreman and D. Posselt, Eur. Phys. J. E, 2000, 1, 87-91.

24 R. Zhang, W. Sun, S. Traistram-Nagle, R. L. Headrick, R. M. Suter and J. F. Nagle, Phys. Rev. Lett., 1995, 74, 2832-2835.

25 G. Pabst, J. Katsaras and V. A. Raghunathan, Phys. Rev. Lett., 2002, 12, art. no. 128101.

26 J. F. Nagle and S. Tristram-Nagle, Curr. Opin. Struct. Biol., 2000, 10, 474- 480

27 K. Sengupta, V. A. Raghunathan and Y. Hatwalne, Phys. Rev. Lett., 2001, 87, 55705.

28 M. Rappolt, G. Pabst, G. Rapp, M. Kriechbaum, H. Amenitsch, C. Krenn, S. Bernstorff and P. Laggner, Eur. Biophys. J., 2000, 29, $125-133$.

29 J. Fournier, Europhys. Lett., 1998, 43, 725-730. 